\title{
Morality of the Heart: Heart Rate Variability and Moral Rule Adherence in Men
}

\author{
Alexander Lischke1,2*, Matthias Weippert ${ }^{3}$, Anett Mau-Moeller ${ }^{3}$ and Rike Pahnke ${ }^{3 *}$ \\ ${ }^{1}$ Department of Psychology, Medical School Hamburg, Hamburg, Germany, ${ }^{2}$ Department of Psychology, University \\ of Greifswald, Greifswald, Germany, ${ }^{3}$ Department of Sport Science, University of Rostock, Rostock, Germany
}

\section{OPEN ACCESS}

Edited by: Julian F. Thayer,

The Ohio State University,

United States

Reviewed by:

DeWayne P. Williams,

University of California, Irvine,

United States

Gewnhi Park,

Hope College, United States

${ }^{*}$ Correspondence:

Alexander Lischke

alexander.lischke@

medicalschool-hamburg.de

Rike Pahnke

rike.pahnke@gmx.de

Specialty section:

This article was submitted to

Autonomic Neuroscience,

a section of the journal

Frontiers in Neuroscience

Received: 30 September 2020

Accepted: 25 June 2021

Published: 07 September 2021

Citation:

Lischke A, Weippert M Mau-Moeller A and Pahnke R (2021) Morality of the Heart: Heart Rate Variability and Moral Rule Adherence in Men. Front. Neurosci. 15:612712. doi: 10.3389/fnins.2021.612712
Moral rules are a cornerstone of many societies. Most moral rules are concerned with the welfare of other individuals, reflecting individuals' innate aversion against harming other individuals. Harming others is associated with aversive experiences, implying that individuals who are sensitive to the aversiveness of these experiences are more likely to follow moral rules than individuals who are insensitive to the aversiveness of these experiences. Individuals' sensitivity for aversive experiences depends on individuals' ability to integrate the underlying neural and physiological processes: Individuals who are more efficient in integrating these processes are more sensitive to the aversiveness that is associated with moral rule violations than individuals who are less efficient in integrating these processes. Individuals who differ in their ability to integrate these processes may, thus, also differ in their inclination to follow moral rules. We tested this assumption in a sample of healthy individuals (67 males) who completed measures of moral rule adherence and integration abilities. Moral rule adherence was assessed with self-report measure and integration abilities were assessed with a resting state measure of heart rate variability (HRV), which reflects prefrontal-(para-)limbic engagement during the integration of physical and neural processes. We found a positive association between individuals' HRV and individuals' moral rule adherence, implying that individuals with efficient integration abilities were more inclined to follow moral rules than individuals with inefficient integration abilities. Our findings support the assumption that individuals with different integration abilities also differ in moral rule adherence, presumably because of differences in aversiveness sensitivity.

Keywords: moral reasoning, moral decision making, idealism, harm avoidance, vagal tone

\section{INTRODUCTION}

Across industrialized and non-industrialized societies, individuals share a common set of moral rules. Most moral rules are concerned with the welfare of others (Haidt and Joseph, 2004), which may be a reflection of individuals' innate aversion against harming others (Hamlin et al., 2007, 2010). Regardless whether individuals imagine or perform harmful actions, they experience aversive emotional reactions that are accompanied by corresponding processes on the neural and physiological level (Cushman et al., 2012; Decety et al., 2012). The aversiveness of these experiences depends on individuals' ability to integrate the corresponding neural and physiological processes (Critchley, 2005). Individuals who are efficient in integrating these processes are more 
sensitive to the aversiveness of harmful actions than individuals who are inefficient in integrating these processes (Cushman et al., 2012; Decety et al., 2012). As a consequence, individuals with efficient integration abilities are more motivated to avoid aversive experiences that are associated with moral rule violations than individuals with inefficient integration abilities (Decety and Cowell, 2014), implying more moral rule adherence in individuals with efficient than inefficient integration abilities.

Individuals' integration abilities can be differentiated on the basis of individuals' heart rate variability (HRV), which serves as a proxy for the interplay of prefrontal and (para-)limbic brain regions during the integration of neural and physiological processes (Smith et al., 2017). Individuals with higher HRV are generally more efficient in integrating these processes than individuals with lower HRV, implying that individuals with higher HRV are also more efficient in integrating neural and physiological processes that are associated with aversive experiences. These differences in integration abilities may render individuals with higher HRV more sensitive to the aversiveness of others' harm than individuals with lower HRV. Individuals with higher HRV are, in fact, more responsive to others' needs and more concerned with others' welfare than individuals with lower HRV (Kogan et al., 2014; Stellar et al., 2015; Lischke et al., 2018a), presumably because individuals with higher HRV are more empathetic and less alexithymic than individuals with lower HRV (Lischke et al., 2017, Lischke et al., 2018b). Due to these differences in aversiveness sensitivity, individuals with higher HRV may be more inclined to follow moral rules than individuals with lower HRV.

Following this notion, we performed an exploratory study where we investigated the association between HRV and moral rule adherence in a sample of healthy individuals. In the absence of previous studies on HRV and moral rule adherence, we felt obliged to provide a concise description rather than a complex explanation of the association between HRV and moral rule adherence. We, thus, tested whether HRV was associated with moral rule adherence and refrained from testing whether this association would be moderated or mediated by aversiveness sensitivity. This allowed us to analyze the association between HRV and moral rule adherence in a clear and simple manner, thereby avoiding issues arising from the use of more complex analyses (e.g., overfitting in structural equation models).

As we were interested to investigate the association between HRV and moral rule adherence in a clear and simple manner, we made arrangements to reduce the complexity of the study design. Given that male and female individuals differ in moral rule adherence and HRV (Abhishekh et al., 2013; Friesdorf et al., 2015), we only included male individuals in our investigation. We, thus, did not have to control for sex- or menstrual cyclerelated differences in moral rule adherence and HRV in our analyses. To reduce the number of possible analyses to a minimum, we used a limited set of measures for the assessment of moral rule adherence and HRV. HRV was assessed with a resting state measure of high-frequency HRV (HF-HRV). HFHRV measures the integration of neurophysiological processes that are associated with an empathic reaction to others' harm (Kogan et al., 2014; Stellar et al., 2015; Lischke et al., 2018b), implying that this may also be the case during violations of moral rules that are concerned with others' welfare. Moral rule adherence was assessed with a self-report measure that differentiated between moral idealism and moral relativism (Forsyth, 1980). Whereas moral idealism is characterized by strict rule following that precludes the violation of moral rules, moral relativism is characterized by flexible rule following that allows the violation of moral rules (Forsyth, 1980). Given that moral idealism reflects moral rule following to a greater extent than moral relativism, we expected individuals' HRV to be associated with individuals' moral idealism rather than with individuals' moral relativism.

\section{MATERIALS AND METHODS}

\section{Participants}

According to an a priori power analysis with $\mathrm{G}^{*}$ Power $^{1}$, we had to investigate a minimum of 67 individuals to be able to detect medium- to large-sized associations between HRV and moral rule adherence in a series of dimensional and categorical analyses $\left[1-\beta=0.80, \alpha=0.05, f^{2}=0.20\right.$, and $f=0.35]$. In order to be included in the study, individuals had to be males with an age range of 18 to 35 years. Individuals who were in psychotherapeutic or psychopharmacological treatment were excluded from the study. Inclusion and exclusion of individuals was determined on the basis of an in-house interview that assessed individuals' demographic (age and sex), anthropometric (height and weight), and health (physical activity, psychotherapeutic treatment, and psychopharmacological treatment) characteristics. Of the 67 individuals who had been recruited for the study, 3 individuals had to be excluded because they were in psychotherapeutic treatment. The final sample, thus, comprised 64 instead of 67 individuals (see Table 1). However, all individuals had provided written informed consent to the study protocol that was approved by the ethics committee of the University of Rostock and that was carried out in accordance with the Declaration of Helsinki.

\section{Procedure}

We followed an established procedure that has been described in more detail elsewhere (Lischke et al., 2017, Lischke et al., 2018a,b). At the beginning of the experimental session, individuals were asked to use the bathroom to control for the effects of bladder filling and gastric distension on individuals' HRV. Thereafter, individuals were seated in a comfortable chair and prepared for a 5-min lasting resting state heart rate $(\mathrm{HR})$ recording. Individuals' $H R$ was recorded with a polar watch (RS800, Polar Electro, Oy, Kempele, Finland) that allowed an accurate assessment of consecutive changes in heartbeats. The consecutive changes in heartbeats were later used for the determination of individuals' HRV. During the HR recording, individuals were instructed to sit still, to breathe spontaneously, and to keep their eyes open. After the HR recording, individuals had to complete a self-report

\footnotetext{
${ }^{1}$ http://www.gpower.hhu.de
} 
TABLE 1 | Sample characteristics.

\begin{tabular}{lcc}
\hline & $\boldsymbol{M}$ & SEM \\
\hline Age (years) & 23.91 & 0.49 \\
Body mass index $\left(\mathrm{kg} / \mathrm{m}^{2}\right)$ & 23.76 & 0.28 \\
Activity (h/week) & 7.66 & 0.49 \\
Moral relativism (EPQ-IDE) & 5.98 & 0.11 \\
Moral idealism (EPQ-REL) & 5.68 & 0.14 \\
Heart rate variability (HF-HRV, ms $\left.{ }^{2}\right)^{a}$ & 2.73 & 0.05 \\
Heart rate variability (RMSSD, ms) & 1.59 & 0.02 \\
\hline
\end{tabular}

EPQ-REL, Ethical Position Questionnaire-Moral relativism (Forsyth, 1980); EPQIDE, Ethical Position Questionnaire-Moral idealism (Forsyth, 1980); HF-HRV, (logtransformed) high-frequency heart rate variability (Shaffer and Ginsberg, 2017); and RMSSD, (log-transformed) root mean square of successive differences between consecutive heart beats (Shaffer and Ginsberg, 2017).

a Data were missing for one participant due to a recording error.

measure of moral rule adherence (Ethical Position Questionnaire, EPQ; Forsyth, 1980). At the end of the experimental session, individuals were debriefed and dismissed.

\section{Heart Rate Variability}

Kubios HRV 2.2 (Tarvainen et al., 2014) was used to determine individuals' HRV on the basis of the HR recordings. The HR recordings were detrended (smoothn priors: $\lambda=500$ ) and, if necessary, artifact corrected (adaptive filtering: cubic spine interpolation) before they were subjected to a spectral analysis (Fast Fourier Transformation) and a time domain analysis. The spectral analysis was used to determine the HRV measure of interest: HF-HRV $(0.15-0.4 \mathrm{~Hz})$. HF-HRV was the HRV measure of interest because HF-HRV tracks the integration of neurophysiological processes that are associated with an empathic reaction to others' harm (Kogan et al., 2014; Stellar et al., 2015; Lischke et al., 2018b), indicating that HF-HRV reflects aversive reactions to violations of others' welfare (see Supplementary Material 1). The time domain analysis was used for the determination of a HRV measure that tracks similar processes as HF-HRV (Shaffer and Ginsberg, 2017): the root mean square of successive differences between consecutive heart beats (RMSSD). This HRV measure was used to determine whether possible associations between HF-HRV and moral rule adherence would generalize across different HRV measures.

\section{Moral Rule Adherence}

The EPQ (Forsyth, 1980) was used to determine individuals' moral rule adherence in terms of moral idealism and moral relativism. The self-report measure comprises 20 items, 10 items that assess moral idealism $[\alpha=0.64]$ and 10 items that assess moral relativism $[\alpha=0.73]$. Whereas moral idealism refers to moral rule adherence in terms of strict rule following that precludes the violation of moral rules in all circumstances, moral relativism refers to moral rule adherence in terms of flexible rule following that allows the violation of moral rules in some circumstances. Given that moral rule adherence is motivated by the concern about others' welfare (Haidt and Joseph, 2004), it is not surprising that moral idealism (i.e., strict rule following) rather than moral relativism (i.e., flexible rule following) is associated with aversive reactions to violations of others' welfare (see Supplementary Material 2).

\section{Statistical Analysis}

In line with recent recommendations (Laborde et al., 2017), dimensional and categorical analyses were performed to investigate the association between HRV and moral rule adherence in the sample of individuals. Combining dimensional and categorical analyses allowed a cross-validation of the respective findings, thereby providing a robustness check for any conclusions that were based on the findings of a particular analysis. For the dimensional analysis, hierarchical regression analyses were run to investigate whether HRV was associated with moral rule adherence among all individuals. For the categorical analyses, analyses of covariance (ANCOVAs) were run to investigate whether moral rule adherence differed between individuals who had been assigned to a high and low HRV group on the basis of a median-split. For both types of analyses, HRV was log-transformed (log 10 ) to account for deviations from normality. Age, body mass index, and physical activity were under statistical control in these analyses because these characteristics may affect the association between individuals' HRV and individuals' moral rule adherence (De Meersman, 1993; Abhishekh et al., 2013; Koenig et al., 2014). To facilitate the interpretation of the analyses (Cohen, 1992; Cumming, 2014), significance values $(p)$ and effect size measures $\left(\eta^{2}, B, R^{2}\right.$, and $\Delta R^{2}$ ) were determined. All analyses were performed with SPSS 24 (SPSS Inc., Chicago, IL, United States).

\section{RESULTS}

\section{Association Between Moral Relativism (EPQ-REL) and Heart Rate Variability (HF-HRV)}

A hierarchical regression analysis was run to investigate the association between HF-HRV and moral relativism among all individuals. Entering individuals' age, body mass index, and physical activity in a first step into the regression model did not explain any variance in individuals' moral relativism $\left[R^{2}=0.02\right.$, $F(3,59)=0.49$, and $p=0.693$; see Table 2]. Age, body mass index, and physical activity were not associated with moral relativism [all $B \leq|0.05|$, all $t(59) \leq|1.20|$, and all $p \geq 0.233$; see Table 2]. Entering individuals' HF-HRV in a second step into the regression model also explained no variance in moral relativism $\left[\Delta R^{2}=0.01, \Delta F(1,58)=0.38\right.$, and $p=0.538$; see Table 2]. HFHRV was, similar to age, body mass index, and physical activity [all $B \leq|0.04|$, all $t(58) \leq|1.07|$, and all $p \geq 0.287$; see Table 2], not associated with moral relativism $[B=0.24, t(58)=0.62$, and $p=0.538$; see Table 2 and Figure 1]. A subsequent ANCOVA revealed that moral relativism was equally pronounced among individuals with higher and lower HF-HRV $[F(1,58)=0.31$, $p=0.581$, and $\eta^{2}=0.005$; see Figure 2], thereby confirming the absence of an association between individuals' HF-HRV and individuals' moral relativism. Repeating the analyses with RMSSD instead of HF-HRV revealed exactly the same findings 
TABLE 2 | Association of moral idealism (EPQ-IDE) or moral relativism (EPQ-REL) with heart rate variability (HF-HRV).

\begin{tabular}{|c|c|c|c|c|c|c|c|c|c|}
\hline \multirow[b]{2}{*}{ Model 1} & \multicolumn{4}{|c|}{ Moral idealism (EPQ-IDE) } & \multirow[b]{2}{*}{ Model 2} & \multicolumn{4}{|c|}{ Moral relativism (EPQ-REL) } \\
\hline & $B$ & SE B & $t$ & $p$ & & $B$ & SE B & $t$ & $p$ \\
\hline Step 1 & & & & & Step 1 & & & & \\
\hline Age (years) & -0.01 & 0.04 & -0.34 & 0.738 & Age (years) & 0.05 & 0.04 & 1.20 & 0.233 \\
\hline Body mass index $\left(\mathrm{kg} / \mathrm{m}^{2}\right)$ & 0.00 & 0.05 & -0.07 & 0.947 & Body mass index $\left(\mathrm{kg} / \mathrm{m}^{2}\right)$ & -0.04 & 0.08 & -0.54 & 0.595 \\
\hline Activity (h/week) & -0.01 & 0.03 & -0.39 & 0.699 & Activity (h/week) & 0.01 & 0.04 & 0.29 & 0.776 \\
\hline Step 2 & & & & & Step 2 & & & & \\
\hline Age (years) & -0.02 & 0.04 & -0.70 & 0.486 & Age (years) & 0.04 & 0.04 & 1.07 & 0.287 \\
\hline Body mass index $\left(\mathrm{kg} / \mathrm{m}^{2}\right)$ & 0.03 & 0.05 & 0.45 & 0.655 & Body mass index $\left(\mathrm{kg} / \mathrm{m}^{2}\right)$ & -0.03 & 0.08 & -0.37 & 0.717 \\
\hline Activity (h/week) & -0.03 & 0.03 & -0.95 & 0.347 & Activity (h/week) & 0.00 & 0.04 & 0.11 & 0.913 \\
\hline Heart rate variability $\left(\mathrm{HF}-\mathrm{HRV}, \mathrm{ms}^{2}\right)^{a}$ & 0.66 & 0.33 & 2.12 & $0.039^{\star}$ & Heart rate variability $\left(\mathrm{HF}-\mathrm{HRV}, \mathrm{ms}^{2}\right)^{a}$ & 0.24 & 0.44 & 0.62 & 0.538 \\
\hline
\end{tabular}

Model 1: step 1: $R^{2}=0.01, F(3,59)=0.10, p=0.959$, step 2: $\Delta R^{2}=0.07, \Delta F(1,58)=4.48, p=0.039^{*} ;$ model 2: step 1: $R^{2}=0.02, F(3,59)=0.49, p=0.693$, step 2: $\Delta R^{2}=0.01, \Delta F(1,58)=0.38, p=0.538$.

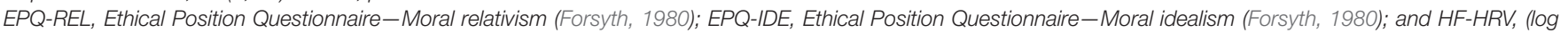
transformed) high-frequency heart rate variability (Shaffer and Ginsberg, 2017).

a Data were missing for one participant due to a recording error.

$* p \leq 0.05$.
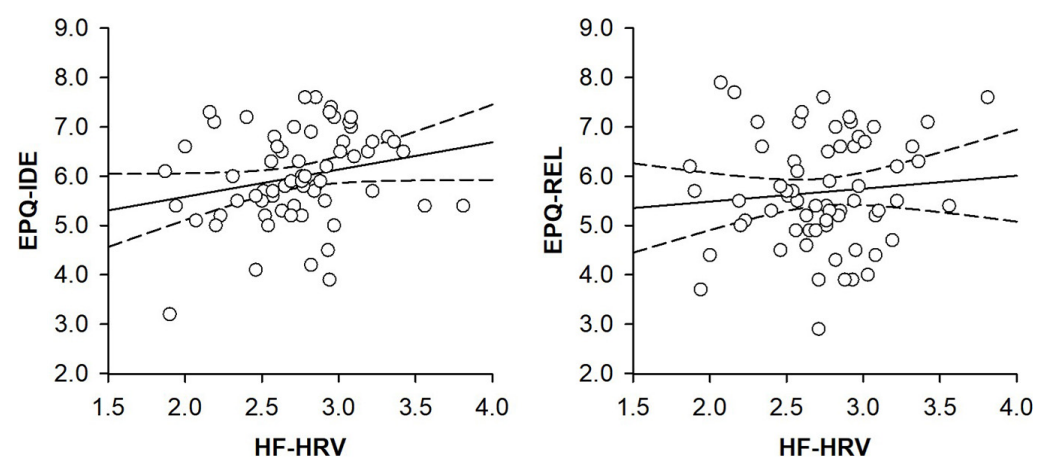

FIGURE 1 | Scatter plots with lines of best fit and 95\% confidence intervals demonstrating associations between (log-transformed) heart rate variability (HF-HRV) and moral idealism (EPQ-IDE) or moral relativism (EPQ-REL) among all individuals.

(see Supplementary Material 3), indicating that the findings generalize across different HRV measures (HF-HRV, RMSSD).

\section{Association Between Moral Idealism (EPQ-IDE) and Heart Rate Variability (HF-HRV)}

Another hierarchical regression analysis was run to investigate the association between HF-HRV and moral idealism among all individuals. Entering individuals' age, body mass index, and physical activity in a first step into the regression model explained no variance in individuals' moral idealism $\left[R^{2}=0.01, F(3\right.$, $59)=0.10$, and $p=0.959$; see Table 2]. Age, body mass index and physical activity were not associated with moral idealism [all $B \leq|-0.01|$, all $t(59) \leq|-0.39|$, and all $p \geq 0.699$; see Table 2]. Entering individuals' HF-HRV in a second step into the regression model explained $7 \%$ of the variance in individuals' moral idealism $\left[\Delta R^{2}=0.07, \Delta F(1,58)=4.48\right.$, and $p=0.039$; see Table 2]. Whereas age, body mass index, and physical activity remained to be unassociated with moral idealism [all $B \leq|-0.03|$, all $t(58) \leq|-0.95|$, and all $p \geq 0.347$; see Table 2], HF-HRV turned out to be associated with moral idealism $[B=0.66, t(58)=2.12$, and $p=0.039$; see Table 2 and Figure 1]. A subsequent ANCOVA showed that moral idealism was more pronounced among individuals with higher than lower HF-HRV $[F(1,58)=5.11, p=0.028$, and $\eta^{2}=0.081$, see Figure 2], thereby confirming the existence of a positive association between individuals' HF-HRV and individuals' moral idealism. The positive association between individuals' HF-HRV and individuals' moral idealism remained unchanged when controlling for individuals' moral realism in the analyses $\left[\Delta R^{2}=0.07, \Delta F(1,57)=4.16, p=0.046 ; B=0.65\right.$, $t(57)=2.04$, and $p=0.046$; see Table 3]. Repeating the analyses with RMSSD instead of HF-HRV revealed similar findings (see Supplementary Material 4), indicating that the findings generalize across different HRV measures (HF-HRV, RMSSD).

\section{DISCUSSION}

In the present study, we investigated whether individuals' ability to integrate neural and physiological processes was associated 


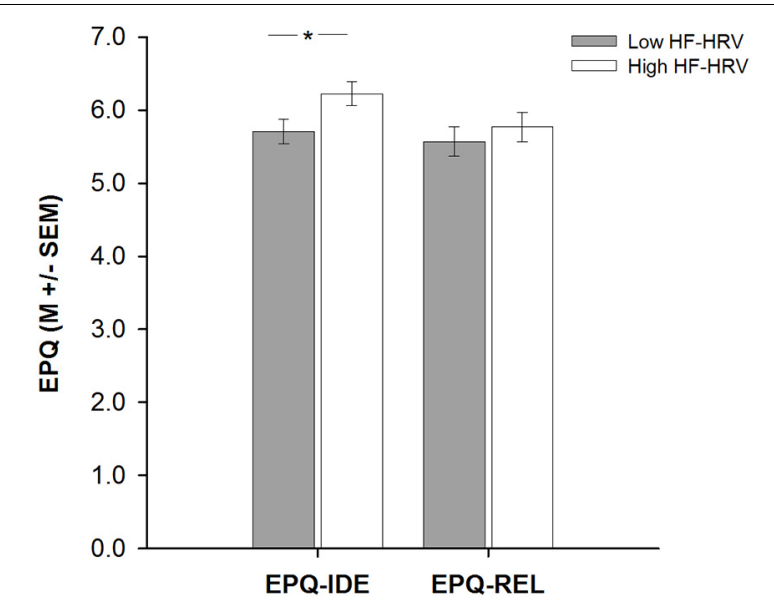

FIGURE 2 | Bar plots demonstrating differences in moral idealism (EPQ-IDE) or moral relativism (EPQ-REL) between individuals with lower and higher heart rate variability (HF-HRV). Bars represent $\mathrm{M} \pm \mathrm{SEM}$. ${ }^{*} P \leq 0.05$.

TABLE 3 | Association of moral idealism (EPQ-IDE) with heart rate variability (HF-HRV) under control of moral realism (EPQ-REL).

\begin{tabular}{lcccc}
\hline & \multicolumn{4}{c}{ Moral Idealism (EPQ-IDE) } \\
\cline { 2 - 5 } Model 1 & B & SE B & $t$ & $p$ \\
\hline Step 1 & & & & \\
Age (years) & -0.02 & 0.04 & -0.47 & 0.640 \\
Body mass index (kg/m²) & 0.00 & 0.06 & 0.00 & 0.997 \\
Activity (h/week) & -0.01 & 0.03 & -0.42 & 0.675 \\
Moral relativism (EPQ-REL) & 0.10 & 0.11 & 0.90 & 0.373 \\
Step 2 & & & & \\
Age (years) & -0.03 & 0.04 & -0.80 & 0.428 \\
Body mass index (kg/m²) & 0.03 & 0.06 & 0.48 & 0.631 \\
Activity (h/week) & -0.03 & 0.04 & -0.96 & 0.343 \\
Moral relativism (EPQ-REL) & 0.08 & 0.11 & 0.75 & 0.454 \\
Heart rate variability (HF-HRV, ms $\left.{ }^{2}\right)^{a}$ & 0.65 & 0.35 & 2.04 & $0.046 *$ \\
\hline
\end{tabular}

Note. Model 1: step 1: $R^{2}=0.02, F(3,58)=0.28, p=0.892$, step 2: $\Delta R^{2}=0.07$, $\Delta F(1,57)=4.16, p=0.046^{*}$.

EPQ-REL, Ethical Position Questionnaire-Moral relativism (Forsyth, 1980); EPQ-IDE, Ethical Position Questionnaire-Moral idealism (Forsyth, 1980); and HF-HRV, (log-transformed) high-frequency heart rate variability (Shaffer and Ginsberg, 2017).

a Data were missing for one participant due to a recording error.

$* p \leq 0.05$.

with individuals' tendency to follow moral rules. Individuals' integration abilities were determined on the basis of a HRV measure that served as a proxy for the interplay of prefrontal and (para-)limbic brain regions during the regulation of neural and physiological processes (Smith et al., 2017). Individuals' tendency to follow moral rules was assessed with a selfreport measure that differentiated between moral idealism and moral relativism (Forsyth, 1980). Applying these measures to a sample of healthy individuals, we found an association between HRV and moral idealism but no association between HRV and moral relativism: moral idealism was more pronounced among individuals with higher than lower HRV, whereas moral relativism was equally pronounced among individuals with higher and lower HRV. These findings emerged in a series of complementary analyses, which helped to ascertain the robustness of the observed associations. To understand these associations, we have to consider that moral idealism and moral relativism refer to distinct but overlapping aspects of moral rule following. Whereas moral idealism refers to strict rule following that precludes the violation of moral rules in all circumstances, moral relativism refers to flexible rule following that allows the violation of moral rules in some circumstances. We considered the conceptual overlap of moral idealism and moral relativism in our analyses and still found an association between moral idealism and HRV. Our findings, thus, show that individuals with higher HRV follow moral rules to a greater extent (i.e., in all circumstances) than individuals with lower HRV. Given that differences in HRV reflect differences in neurophysiological integration (Smith et al., 2017), our findings support the assumption that individuals with higher integration abilities show more moral rule adherence than individuals with lower integration abilities.

Whether individuals with efficient and inefficient integration abilities follow moral rules may depend on their sensitivity for aversive experiences that are associated with real or imagined violations of moral rules (Cushman et al., 2012; Decety et al., 2012). Individuals whose psychological traits render them aversiveness sensitive, like, for example, empathetic individuals (Conway and Gawronski, 2013; Reynolds and Conway, 2018), are more inclined to follow moral rules than individuals whose psychological traits render them aversiveness insensitive, like, for example, alexithymic individuals (Koven, 2011; Patil and Silani, 2014). These differences in moral rule adherence are even more pronounced in individuals who show abnormal representations of these psychological traits, like, for, example, autistic or psychopathic individuals (Koenigs et al., 2012; Patil et al., 2016). However, individuals who differ in empathy, alexithymia, autism, or psychopathy also seem to differ in their ability to integrate neural and physiological processes as suggested by the respective differences in individuals' HRV (Hansen et al., 2007; Kuiper et al., 2017; Lischke et al., 2018b). It may, thus, be possible that differences in individuals' integration abilities contribute to differences in individuals' aversiveness sensitivity that lead to differences in individuals' moral rule adherence. Considering that individuals' HRV reflect differences in individuals' integration abilities (Smith et al., 2017), we assume that individuals with lower HRV showed more moral rule adherence than individuals with higher HRV because of differences in individuals' aversiveness sensitivity for moral rule violations.

Individuals with efficient integration abilities are more successful in engaging prefrontal and (para-)limbic brain regions for the regulation of neural and physiological processes than individuals with inefficient integration abilities (Critchley, 2005), indicating that differences in prefrontal(para-)limbic engagement may account for differences in aversiveness sensitivity between individuals with efficient and inefficient integration abilities. However, differences in 
prefrontal-(para-)limbic engagement may account not only for differences in individuals' aversiveness sensitivity but also for differences in individuals' moral rule adherence because naturally occurring or experimentally induced alterations in these brain regions impair individuals' integration abilities as well as individuals' moral rule adherence (Mendez and Shapira, 2009; Moretto et al., 2010; Tassy et al., 2012). Individuals with abnormal representations of empathy, alexithymia, autism, and psychopathy also show alterations in prefrontal and (para)limbic brain regions that are associated with impairments in aversiveness sensitivity and moral rule adherence (Silani et al., 2008; Decety et al., 2013), indicating that overlapping networks of prefrontal and (para-)limbic brain regions are implicated in the integration of neural and physiological processes that are relevant for the experience of aversiveness in the context of moral rule violations (Decety and Cowell, 2014). The interplay of prefrontal and (para-)limbic brain regions can be assessed on the basis of individuals HRV (Smith et al., 2017), suggesting that differences in individuals' HRV reflect differences in individuals' integration abilities that are due to differences in prefrontal-(para-)limbic engagement. We, thus, assume that individuals with higher HRV showed more moral rule adherence than individuals with lower HRV because individuals with higher HRV were more efficient in engaging prefrontal and (para-)limbic brain regions for the integration of neurophysiological processes that account for the aversiveness of moral rule violations than individuals with lower HRV.

Although these assumptions appear to be plausible, we think that the assumptions have to be validated in further studies that follow a less exploratory approach than the present study. These studies should investigate the association between individuals' HRV and individuals' moral rule adherence with more complex measures and in more diverse samples than the present study. The present study investigated this association in a homogenous sample of male individuals, leaving open whether similar associations would emerge in heterogeneous samples that include male and female individuals. Given that female individuals have a higher HRV and a higher moral rule adherence than male individuals (Abhishekh et al., 2013; Friesdorf et al., 2015), the association between HRV and moral rule adherence may be more pronounced among female than male individuals. Future studies that include male and female individuals may help to determine whether this is the case. These studies should also include male and female individuals with a more diverse background in their investigation to determine whether the proposed associations between individuals' HRV and individuals' moral rule adherence generalize across different populations (e.g., including individuals with different ages or ethnicities). The present study explored this association with a combination of physiological and self-report measures. Although these measures allowed us to describe the association between HRV and moral rule adherence in a concise manner, they did not allow us to provide a complex explanation of this association. Neural, physiological, and behavioral measures that may have helped to provide such an explanation were not employed (e.g., task-based measures of moral rule adherence and aversiveness sensitivity and imaging-based measures of neurophysiological integration). We were, thus, unable to probe the psychological and neurobiological mechanisms underlying the association between HRV and moral rule adherence (e.g., testing the mediating or moderating role of aversiveness sensitivity). These mechanisms may involve the neurophysiological integration of aversive reactions to violations of others' welfare, but whether this is in fact the case remains to be determined. Studies that combine neural, physiological, and behavioral measures in their investigation may be more successful in elucidating the psychological and neurobiological mechanisms of HRV and moral rule adherence than the present study (e.g., combining task-based measures of moral rule adherence and aversiveness sensitivity with imaging-based measures of neurophysiological integration). Studies that manipulate these mechanisms with appropriate methods may help to make causal inferences about the association between HRV and moral rule adherence (e.g., increasing or decreasing HRV with brain stimulation techniques), thereby providing first insights into intervention programs for individuals who have difficulties in moral rule following (e.g., HRV biofeedback training for individuals with psychopathy). We hope that our exploratory study opened an avenue for these types of studies.

To sum up, we found a positive association between HRV and moral rule adherence in a sample of healthy individuals. Individuals with higher HRV showed more moral rule adherence than individuals with lower HRV. We assume that the differences in individuals moral rule adherence were due to differences in individuals' aversiveness sensitivity for moral rule violations that were determined by differences in individuals' prefrontal-(para-)limbic engagement during the integration of neurophysiological processes. As we based these assumptions on the findings of previous studies (Decety and Cowell, 2014; Smith et al., 2017), we encourage researchers to validate these assumptions in further studies. Together, these studies will provide important insights into the psychological and neurobiological mechanisms underlying the association between individuals' HRV and individuals' moral rule adherence. Whether these studies will help to develop treatment interventions for individuals who have difficulties in moral rule adherences has to be seen in the future.

\section{DATA AVAILABILITY STATEMENT}

The datasets presented in this article are not readily available because of ethical restrictions. Requests to access the datasets should be directed to AL.

\section{ETHICS STATEMENT}

The studies involving human participants were reviewed and approved by University of Rostock. The patients/participants provided their written informed consent to participate in this study. 


\section{AUTHOR CONTRIBUTIONS}

$\mathrm{AL}$ and RP designed the study, analyzed the data, and wrote the manuscript. AL, AM-M, and MW collected the data. AM-M and MW contributed to writing, reviewing, and editing of the manuscript. All authors approved the final version of the manuscript.

\section{FUNDING}

Funding for this study was provided by a grant from the German Research Foundation to AL (DFG; LI 2517/2-1). The funding source had no further role in study design, in the collection, analysis, and interpretation of data; in the writing of

\section{REFERENCES}

Abhishekh, H. A., Nisarga, P., Kisan, R., Meghana, A., Chandran, S., Trichur, R., et al. (2013). Influence of age and gender on autonomic regulation of heart. J. Clin. Monit. Comput. 27, 259-264. doi: 10.1007/s10877-012-9424-3

Cohen, J. (1992). A power primer. Psychol. Bull. 112, 155-159.

Conway, P., and Gawronski, B. (2013). Deontological and utilitarian inclinations in moral decision making: a process dissociation approach. J. Pers. Soc. Psychol. 104, 216-235. doi: 10.1037/a0031021

Critchley, H. D. (2005). Neural mechanisms of autonomic, affective, and cognitive integration. J. Comp. Neurol. 493, 154-166. doi: 10.1002/cne.20749

Cumming, G. (2014). The new statistics: why and how. Psychol. Sci. 25, 7-29. doi: $10.1177 / 0956797613504966$

Cushman, F., Gray, K., Gaffey, A., and Mendes, W. B. (2012). Simulating murder: the aversion to harmful action. Emotion 12, 2-7. doi: 10.1037/a0025071

De Meersman, R. E. (1993). Heart rate variability and aerobic fitness. Am. Heart J. 125, 726-731. doi: 10.1016/0002-8703(93)90164-5

Decety, J., Chen, C., Harenski, C., and Kiehl, K. A. (2013). An fMRI study of affective perspective taking in individuals with psychopathy: imagining another in pain does not evoke empathy. Front. Hum. Neurosci. 7:489. doi: 10.3389/ fnhum.2013.00489

Decety, J., and Cowell, J. M. (2014). The complex relation between morality and empathy. Trends Cogn. Sci. 18, 337-339. doi: 10.1016/j.tics.2014.04.008

Decety, J., Michalska, K. J., and Kinzler, K. D. (2012). The contribution of emotion and cognition to moral sensitivity: a neurodevelopmental study. Cereb. Cortex 22, 209-220. doi: 10.1093/cercor/bhr111

Forsyth, D. R. (1980). A taxonomy of ethical ideologies. J. Pers. Soc. Psychol. 39:175. doi: 10.1037/0022-3514.39.1.175

Friesdorf, R., Conway, P., and Gawronski, B. (2015). Gender differences in responses to moral dilemmas: a process dissociation analysis. Pers. Soc. Psychol. Bull. 41, 696-713. doi: 10.1177/0146167215575731

Haidt, J., and Joseph, C. (2004). Intuitive ethics: how innately prepared intuitions generate culturally variable virtues. Daedalus 133, 55-66. doi: 10.1162/ 0011526042365555

Hamlin, J. K., Wynn, K., and Bloom, P. (2007). Social evaluation by preverbal infants. Nature 450, 557-559. doi: 10.1038/nature06288

Hamlin, J. K., Wynn, K., and Bloom, P. (2010). Three-month-olds show a negativity bias in their social evaluations. Dev. Sci. 13, 923-929. doi: 10.1111/j.1467-7687. 2010.00951.x

Hansen, A. L., Johnsen, B. H., Thornton, D., Waage, L., and Thayer, J. F. (2007). Facets of psychopathy, heart rate variability and cognitive function. J. Pers. Disord. 21, 568-582. doi: 10.1521/pedi.2007.21.5.568

Koenig, J., Jarczok, M. N., Warth, M., Ellis, R. J., Bach, C., Hillecke, T. K., et al. (2014). Body mass index is related to autonomic nervous system activity as measured by heart rate variability-a replication using short term measurements. J. Nutr. Health Aging 18, 300-302. doi: 10.1007/s12603-0140022-6 the manuscript; and in the decision to submit the manuscript for publication.

\section{ACKNOWLEDGMENTS}

The authors would like to thank Stephan Lau for valuable comments on an earlier version of the manuscript.

\section{SUPPLEMENTARY MATERIAL}

The Supplementary Material for this article can be found online at: https://www.frontiersin.org/articles/10.3389/fnins. 2021.612712/full\#supplementary-material

Koenigs, M., Kruepke, M., Zeier, J., and Newman, J. P. (2012). Utilitarian moral judgment in psychopathy. Soc. Cogn. Affect. Neurosci. 7, 708-714. doi: 10.1093/ scan/nsr048

Kogan, A., Oveis, C., Carr, E. W., Gruber, J., Mauss, I. B., Shallcross, A., et al. (2014). Vagal activity is quadratically related to prosocial traits, prosocial emotions, and observer perceptions of prosociality. J. Pers. Soc. Psychol. 107, 1051-1063. doi: $10.1037 / \mathrm{a} 0037509$

Koven, N. S. (2011). Specificity of meta-emotion effects on moral decision-making. Emotion 11, 1255-1261. doi: 10.1037/a0025616

Kuiper, M. W. M., Verhoeven, E. W. M., and Geurts, H. M. (2017). Heart rate variability predicts inhibitory control in adults with autism spectrum disorders. Biol. Psychol. 128, 141-152. doi: 10.1016/j.biopsycho.2017.07. 006

Laborde, S., Mosley, E., and Thayer, J. F. (2017). Heart rate variability and cardiac vagal tone in psychophysiological research - Recommendations for experiment planning, data analysis, and data reporting. Front. Psychol. 8:213. doi: 10.3389/ fpsyg.2017.00213

Lischke, A., Lemke, D., Neubert, J., Hamm, A. O., and Lotze, M. (2017). Inter-individual differences in heart rate variability are associated with interindividual differences in mind-reading. Sci. Rep. 7:11557. doi: 10.1038/s41598017-11290-1

Lischke, A., Mau-Moeller, A., Jacksteit, R., Pahnke, R., Hamm, A. O., and Weippert, M. (2018a). Heart rate variability is associated with social value orientation in males but not females. Sci. Rep. 8:7336. doi: 10.1038/s41598-018-25739-4

Lischke, A., Pahnke, R., Mau-Moeller, A., Behrens, M., Grabe, H. J., Freyberger, H. J., et al. (2018b). Inter-individual differences in heart rate variability are associated with inter-individual differences in empathy and alexithymia. Front. Psychol. 9:229. doi: 10.3389/fpsyg.2018.00229

Mendez, M. F., and Shapira, J. S. (2009). Altered emotional morality in frontotemporal dementia. Cogn. Neuropsychiatry 14, 165-179. doi: 10.1080/ 13546800902924122

Moretto, G., Ladavas, E., Mattioli, F., and di Pellegrino, G. (2010). A psychophysiological investigation of moral judgment after ventromedial prefrontal damage. J. Cogn. Neurosci. 22, 1888-1899. doi: 10.1162/jocn.2009. 21367

Patil, I., Melsbach, J., Hennig-Fast, K., and Silani, G. (2016). Divergent roles of autistic and alexithymic traits in utilitarian moral judgments in adults with autism. Sci. Rep. 6:23637. doi: 10.1038/srep23637

Patil, I., and Silani, G. (2014). Reduced empathic concern leads to utilitarian moral judgments in trait alexithymia. Front. Psychol. 5:501. doi: 10.3389/fpsyg.2014. 00501

Reynolds, C. J., and Conway, P. (2018). Not just bad actions: affective concern for bad outcomes contributes to moral condemnation of harm in moral dilemmas. Emotion 18, 1009-1023. doi: 10.1037/emo0000413

Shaffer, F., and Ginsberg, J. P. (2017). An overview of heart rate variability metrics and norms. Front. Public Health 5:258. doi: 10.3389/fpubh.2017. 00258 
Silani, G., Bird, G., Brindley, R., Singer, T., Frith, C., and Frith, U. (2008). Levels of emotional awareness and autism: an fMRI study. Soc. Neurosci. 3, 97-112. doi: 10.1080/17470910701577020

Smith, R., Thayer, J. F., Khalsa, S. S., and Lane, R. D. (2017). The hierarchical basis of neurovisceral integration. Neurosci. Biobehav. Rev. 75, 274-296. doi: 10.1016/j.neubiorev.2017.02.003

Stellar, J. E., Cohen, A., Oveis, C., and Keltner, D. (2015). Affective and physiological responses to the suffering of others: compassion and vagal activity. J. Pers. Soc. Psychol. 108, 572-585. doi: 10.1037/pspi00 00010

Tarvainen, M. P., Niskanen, J. P., Lipponen, J. A., Ranta-Aho, P. O., and Karjalainen, P. A. (2014). Kubios HRV-heart rate variability analysis software. Comput. Methods Programs Biomed. 113, 210-220. doi: 10.1016/j.cmpb.2013. 07.024

Tassy, S., Oullier, O., Duclos, Y., Coulon, O., Mancini, J., Deruelle, C., et al. (2012). Disrupting the right prefrontal cortex alters moral judgement. Soc. Cogn. Affect. Neurosci. 7, 282-288. doi: 10.1093/scan/nsr008
Conflict of Interest: The authors declare that the research was conducted in the absence of any commercial or financial relationships that could be construed as a potential conflict of interest.

Publisher's Note: All claims expressed in this article are solely those of the authors and do not necessarily represent those of their affiliated organizations, or those of the publisher, the editors and the reviewers. Any product that may be evaluated in this article, or claim that may be made by its manufacturer, is not guaranteed or endorsed by the publisher.

Copyright (c) 2021 Lischke, Weippert, Mau-Moeller and Pahnke. This is an openaccess article distributed under the terms of the Creative Commons Attribution License (CC BY). The use, distribution or reproduction in other forums is permitted, provided the original author(s) and the copyright owner(s) are credited and that the original publication in this journal is cited, in accordance with accepted academic practice. No use, distribution or reproduction is permitted which does not comply with these terms. 